\title{
Performance of WIBA Energy Detector in Rural and Remote Area Channel
}

\author{
Johanna Vartiainen \\ Centre for Wireless Communications \\ University of Oulu \\ Oulu, Finland \\ johanna.vartiainen@oulu.fi \\ Luciano Mendes \\ Radiocommunications Research Center \\ Inatel \\ Brazil \\ luciano@inatel.br
}

\author{
Marja Matinmikko-Blue \\ Centre for Wireless Communications \\ University of Oulu \\ Oulu, Finland \\ marja.matinmikko@oulu.fi
}

\author{
Alexandre Matos \\ Federal University of Ceará \\ Brazil \\ alexandrematos@gtel.ufc.br
}

\author{
Heikki Karvonen \\ Centre for Wireless Communications \\ University of Oulu \\ Oulu, Finland \\ heikki.karvonen@oulu.fi
}

\author{
Carlos Silva \\ Federal University of Ceará \\ Brazil \\ cfms@gtel.ufc.br
}

\begin{abstract}
Connectivity in low-density rural and remote areas where distances are long is a big challenge because of high deployment costs and challenging radio channels with long delay profiles. Spectrum sharing can make spectrum available for 5G local network deployments to serve rural and remote areas. Spectrum sensing can be used to complement the traditional database approach in order to enable efficient and dynamic use of the radio spectrum. In rural and remote areas, long range coverage is required in order to enable flexible and cost-effective solutions. This calls for efficient and low-complex sensing methods who are able to operate in those challenging environments. In this paper we study spectrum sensing method called the windowbased (WIBA) energy detector in a challenging rural area channel model for 5G networks. The results are compared to that of the localization algorithm based on double-thresholding (LAD) energy detector. Simulations using a rural area channel model with long delay profile indicated that the WIBA method is able to operate in a rural area channel, and it clearly outperforms the LAD method in terms of detection distance. The detection difference was even 15-fold for the WIBA method, depending on the transmit power and the signal bandwidth.
\end{abstract}

Index Terms-spectrum sensing, signal detection, rural area, channel model.

\section{INTRODUCTION}

The exploitation of TVWSs (Television White Spaces) [1], using dynamic and fragmented spectrum allocation (DSA) [2] controlled by a cognitive engine [3], is a key feature for remote and rural area wireless $5 \mathrm{G}$ networks. This innovative and flexible use of spectrum is not allowed to harm the incumbent spectrum users, which have priority over the spectrum. Initial propositions for TVWS exploitation have relied on a geolocation database [4] that store all technical and geographic information about the TV stations and use a

This research has received funding from the European Union Horizon 2020 Programme (H2020/2017-2019) under grant agreement N0. 777137 and from the Ministry of Science, Technology under the 4th EU-BR Coordinated Call Information and Communication Technologies through 5G-RANGE project. In addition, this research has been financially supported in part by Academy of Finland 6Genesis Flagship (grant 318927). propagation prediction algorithm to define which channels can be used in a given geographic position.

Relying only on the geolocation database is not enough to provide the necessary protection for the primary users or to fully exploit the vacant spectrum for two main reasons. The first one is related to the fact that the coverage prediction algorithms do not consider all propagation mechanisms, which means that areas considered to be uncovered can indeed be reached by the primary transmitter signal, resulting in performance degradation for the secondary usage. The second reason is related to potential unauthorized transmissions which cannot be prevented in all cases. For example, pirate TV stations which are as a problem in some countries do not have their data inserted in the geolocation database. Although these broadcasting signals do not need to be protected, they can hinder the operation of the secondary networks running in the same channel, which would be considered available by the geolocation database. Therefore, spectrum sensing performed by the secondary networks is essential for providing the appropriate protection of the secondary user for the incumbents. In this case, each a secondary node measures the spectrum at its geographic position and performs a spectrum sensing algorithm to determine if the channel is available or occupied. This decision can be combined with the geolocation database to improve the protection of the primary users and increase the exploitation of all spectrum opportunities.

In this paper, a wide-band window-based spectrum sensing technique called the WIBA [5] energy detector (ED) is proposed to be used for the detection of signals in remote area scenario. Unlike conventional energy detectors, the WIBA ED can detect signals also below the noise level. This increases the detection distance between the transmitter and the receiver, that is very important in rural and remote areas. The performance of the WIBA ED is evaluated in terms of determining the achievable detection distance. This is the first case when the recently proposed WIBA ED is studied in other 


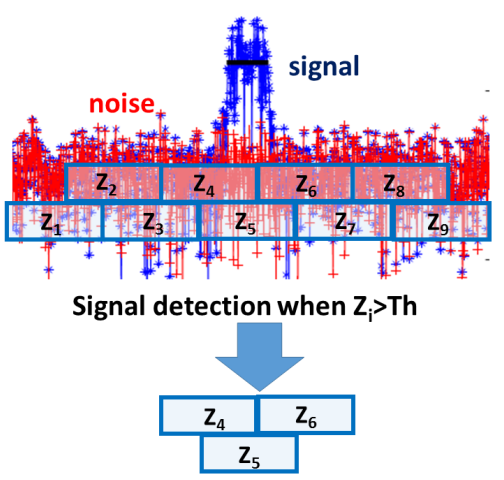

Fig. 2. The WIBA method operation principle.

where threshold parameter $T$ comes from [9], [10]

$$
P_{\mathrm{FA}}=e^{-T M} \sum_{k=0}^{M-1} \frac{1}{k !}(T M)^{k}
$$

where $P_{\mathrm{FA}}$ is the desired false alarm rate. The $P_{\mathrm{FA}}$ defines how many samples are above the threshold if there is only a noise present. For example, if $N=1024$ and $P_{\mathrm{FA}}=0.01(=1 \%), 10$ samples from total of 1024 samples are above the threshold in the noise-only case. The threshold parameter $T$ is constant for specific $M$ and $P_{\mathrm{FA}}$, and is calculated before beforehand and given as an input to WIBA algorithm.

In the performance evaluation, a well-known signal detection method called the LAD method [11] [12] is used as a point of comparison. The LAD method utilizes iterative forward consecutive mean excision (FCME) threshold setting [13]. The threshold setting process corresponds to the WIBA threshold setting when $M=1$ (and $L=N$ ). In that case, the threshold parameter can be derived from (5) as

$$
T_{\mathrm{CME}}=-\ln \left(P_{\mathrm{FA}}\right) .
$$

The FCME algorithm rearranges frequency domain samples in an ascending order according to sample energy. The detection threshold is $T_{h}=T_{\mathrm{CME}} \bar{y}$, where $T_{\mathrm{CME}}$ is from (6) and $\bar{y}$ denotes the mean of energy samples. In the first iteration, the mean is calculated from the initial set that contains $10 \%$ of the samples with smallest energy. The samples below the threshold are added to the initial set, and this iteration continues until there are no samples below the threshold. The LAD method uses two FCME thresholds (upper and lower), by using two different threshold parameters. The LAD method clusters adjacent samples above the lower threshold. The cluster is from a signal if at least one of the samples in the cluster is also above the upper threshold. ACC parameter that allows $p$ (usually $p=3$ ) samples [12] to be below the lower threshold between two accepted clusters, is used to increase the detection performance [12].

\section{Simulations}

The detection performance of the WIBA method was studied by Matlab simulations, and the results were compared to

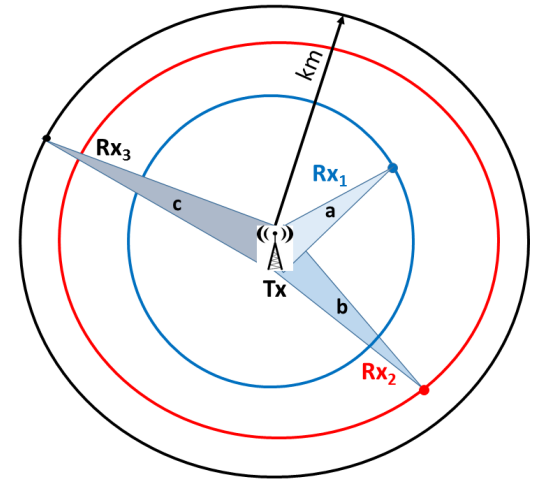

Fig. 3. Detection distance $(\mathrm{Tx}-\mathrm{Rx})$ depends on the transmit signal power, signal BW and the channel.

the LAD method performance. The LAD method is known as a simple and effective ED method. Typical performance requirement for spectrum sensing algorithms is that probability of detection $P_{d}>0.9$ (i.e. 90\%) [14], therefore it is also assumed here. Detection distance in kilometers $(\mathrm{km})$ was of interest in our performance evaluation. In practice, the detection distance corresponds a radius of a circular detection zone as illustrated in Fig. 3. Therein, transmitter Tx transmits three signals $\mathrm{a}, \mathrm{b}$ and $\mathrm{c}$ with different transmit powers and BWs. Figure illustrates the detection distance for three receivers, $\mathrm{Rx}_{1}-\mathrm{Rx}_{3}$, that are able to detect the signals on different distances, depending on the signal power, BW and the propagation channel.

Rural area channel model presented in Section II was used in the simulations and the signal to be detected was a bandlimited raised-cosine binary shift keying (RC-BPSK) modulated signal. This signal was chosen to represent a general telecommunication signal. Note that both the studied methods are robust to signal and modulation types, and also to the frequency areas [5], [12]. Assumed total sensing BW was $23.4 \mathrm{MHz}$ and carrier frequency was $700 \mathrm{MHz}$. There were $N=1024$ frequency domain samples. Transmit power, signal BW and detection window length $M$ varied. Transmitted signal powers were $17-83 \mathrm{dBm}$ and used signal $\mathrm{BW}$ values were $0.9-6 \mathrm{MHz}$ corresponding to $4-25.6 \%$ of the total $\mathrm{BW}$, respectively. Corresponding window lengths $M$ were $44-768$ samples, so that the lengths equal to the signal BW. The number of Monte Carlo iterations was 3000. The WIBA method used $P_{F A}=0.01,50 \%$ overlapping, and $L \approx 2 \frac{N}{M}$. The LAD threshold parameters were $13.81\left(P_{F A}=10^{-6}\right)$ and $2.66\left(P_{F A}=0.07\right)$, and ACC parameter $p=3$.

In the simulations, detection probability $P_{d}$ vs. detection distance between Tx and Rx was studied. In Table II, it is shown what is the maximum distance between $\mathrm{Tx}$ and $\mathrm{Rx}$ for different transmit power and signal BW values so that the detection probability requirement $P_{d} \geq 0.9$ will be met in the WIBA and the LAD methods case. For example, when transmit power is $53 \mathrm{dBm}$ and signal $\mathrm{BW}$ is $2 \mathrm{MHz}$, signal can be detected (detection probability $P_{d} \geq 0.9$ ) when Tx- 
TABLE II

FOR DIFFERENT TRANSMIT POWER [DBM] AND SIGNAL BW VALUES [MHZ], THE MAXIMUM TX-RX DISTANCE WITH A REQUIREMENT THAT DETECTION PROBABILITY $P_{d} \geq 0.9$.

\begin{tabular}{c|c|l|l}
$\begin{array}{c}\text { Transmit power } \\
{[\mathrm{dBm}]}\end{array}$ & $\begin{array}{c}\text { Signal BW } \\
{[\mathrm{MHz}] / \%}\end{array}$ & $\begin{array}{l}\text { Tx-Rx distance } \\
\text { WIBA method }\end{array}$ & $\begin{array}{l}\text { Tx-Rx distance } \\
\text { LAD method }\end{array}$ \\
\hline 83 & $5.85 / 25$ & $>200 \mathrm{~km}$ & $40 \mathrm{~km}$ \\
\hline 53 & $2 / 8.6$ & $<34 \mathrm{~km}$ & $<7 \mathrm{~km}$ \\
53 & $4 / 17.1$ & $<20 \mathrm{~km}$ & $<3 \mathrm{~km}$ \\
53 & $6 / 25.6$ & $<15 \mathrm{~km}$ & $<1 \mathrm{~km}$ \\
\hline 46 & $2 / 8.6$ & $<15 \mathrm{~km}$ & $<6 \mathrm{~km}$ \\
46 & $4 / 17.1$ & $<9 \mathrm{~km}$ & $<3 \mathrm{~km}$ \\
46 & $6 / 25.6$ & $<6 \mathrm{~km}$ & $<1 \mathrm{~km}$ \\
\hline 43 & $5.85 / 25$ & $<4 \mathrm{~km}$ & - \\
\hline 36 & $5.85 / 25$ & $<2 \mathrm{~km}$ & - \\
\hline 30 & $2 / 8.6$ & $<3 \mathrm{~km}$ & - \\
30 & $4 / 17.1$ & $<2 \mathrm{~km}$ & - \\
\hline 30 & $6 / 25.6$ & $<1 \mathrm{~km}$ & - \\
\hline 17 & $0.9 / 4$ & $<900 \mathrm{~m}$ & $<200 \mathrm{~m}$
\end{tabular}

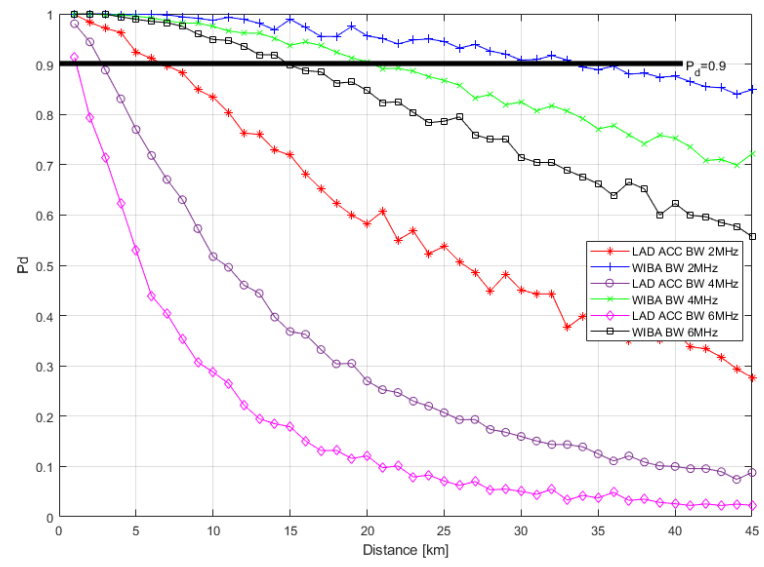

Fig. 4. $P_{d}$ vs. detection distance results when transmit power of the signal is $53 \mathrm{dBm}$. $M=102$ for $3 \mathrm{MHz}$ signal, 180 for $4 \mathrm{MHz}$ signal and 264 for $6 \mathrm{MHz}$ signal.

Rx distance is 34 (WIBA) and 7 (LAD) $\mathrm{km}$ (second line in Table II). The performance difference is remarkable, being almost five-fold. It can be seen that the narrower the signal is, and the higher the transmit power is, the longer is the Tx - Rx distance that the signal can be detected. When transmit power is $83 \mathrm{dBm}$ and signal $\mathrm{BW}$ is $5.85 \mathrm{MHz}(=25 \%)$, the WIBA method is able to detect signal when Tx-Rx distance is even $200 \mathrm{~km}$. Instead, the LAD method is able to detect the signal when Tx-Rx distance is at most $40 \mathrm{~km}$. The WIBA method outperforms the LAD method in all studied cases: it gives $3-15$ times larger detection distances than the LAD method. The wider the signal the bigger the detection distance difference.

In Figs. 4 - 7, transmitted signal power values were $53,46,30$ and $20 \mathrm{dBm}$. While used $\mathrm{BW}$ values of the detected signal were 2,4 or $6 \mathrm{MHz}$ corresponding to $8.6 \%, 17.1 \%$ and $25.6 \%$ of the sensing BW, respectively. Corresponding window lengths were 102,180 and 264 samples, being optimal so that

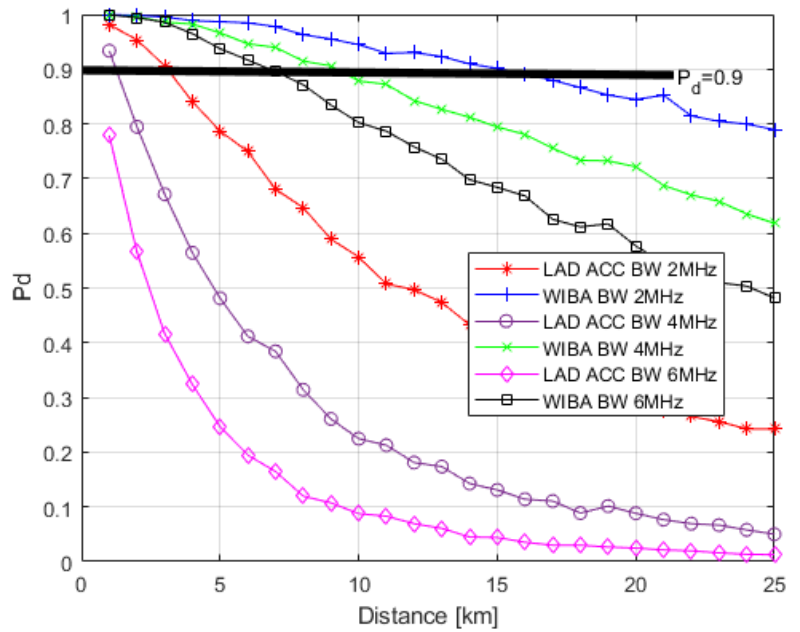

Fig. 5. $\quad P_{d}$ vs. detection distance results when transmit power of the signal is $46 \mathrm{dBm}$. $M=102$ for $3 \mathrm{MHz}$ signal, 180 for $4 \mathrm{MHz}$ signal and 264 for $6 \mathrm{MHz}$ signal.

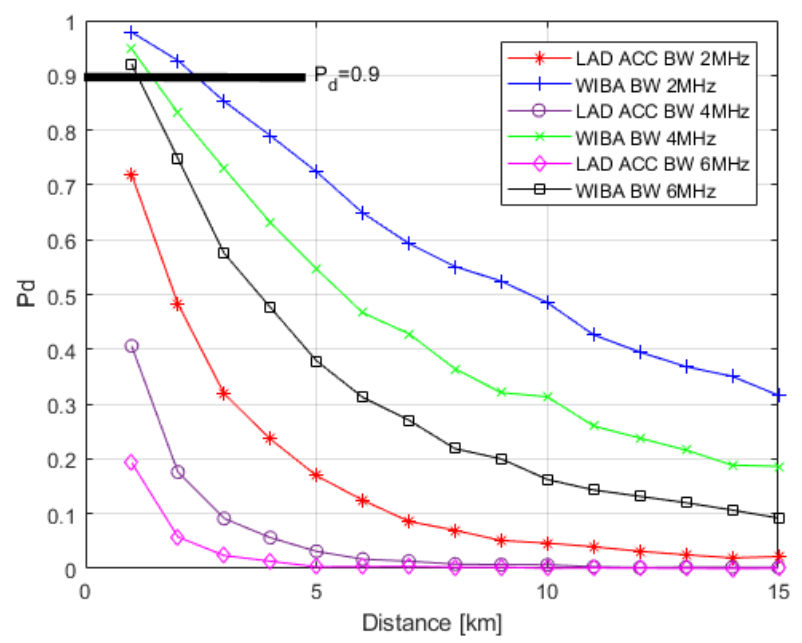

Fig. 6. $P_{d}$ vs. detection distance results when transmit power of the signal is $30 \mathrm{dBm}$. $M=102$ for $3 \mathrm{MHz}$ signal, 180 for $4 \mathrm{MHz}$ signal and 264 for $6 \mathrm{MHz}$ signal.

the lengths equal to the signal $\mathrm{BW}$. The performance target of signal detection was $P_{d} \geq 0.9$, which is marked with the horizontal lines in the pictures. When the WIBA method is used and transmit power is high ( $\geq 46 \mathrm{dBm})$, the curves fall evenly whereas in the LAD method case the curves first fall steeply. From Fig. 4 can be seen that both the methods are able to find all the signals from some distance. However, there is a big difference between the detection distances. For example, when signal BW is $2 \mathrm{MHz}$ and the WIBA method is used, the detection distance is $34 \mathrm{~km}$. When the LAD method is used, the detection distance is $27 \mathrm{~km}$ less, that is, only $7 \mathrm{~km}$. In Fig. 5 case, the WIBA method can detect all the signals, as the LAD method detects only the two narrowest ones (2 and 4 $\mathrm{MHz}$ ). When transmit power is $30 \mathrm{dBm}$, the WIBA method is 


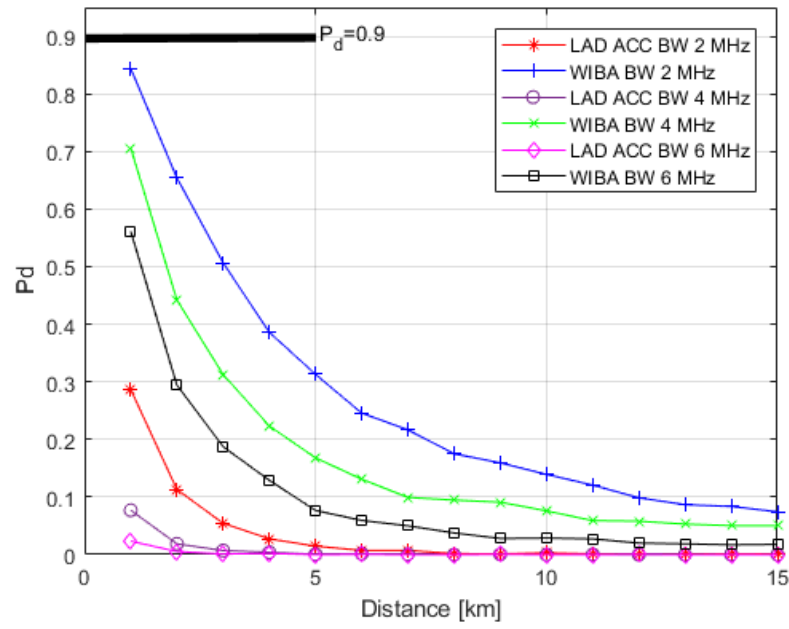

Fig. 7. $P_{d}$ vs. detection distance results when transmit power of the signal is $20 \mathrm{dBm}$.

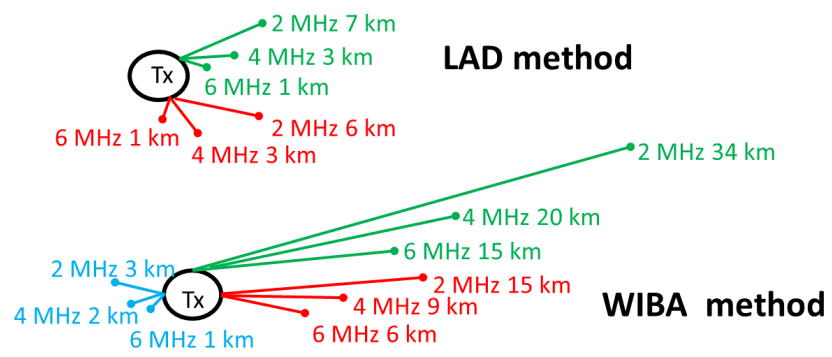

Tx power $53 \mathrm{dBm} 46 \mathrm{dBm} 30 \mathrm{dBm}$

Fig. 8. Distance $[\mathrm{km}]$ where the LAD and WIBA methods can detect the signal at $P_{d} \geq 0.9$. Transmit power is 53,46 and $30 \mathrm{dBm}$ and signal $\mathrm{BW}$ is 2,4 and $6 \mathrm{MHz}$.

able to detect the signals (Fig. 6). However, the LAD method gets at its best $P_{d}=0.7$, so the signals can not be detected using the LAD method. When transmit power is $20 \mathrm{dBm}$, for both the methods, the signal can not be detected at all (Fig. 7). Results for 53, 46 and $30 \mathrm{dBm}$ signals are illustrated in Fig. 8. It can clearly be seen that the WIBA method outperforms the LAD method. The wider the signal and the higher the transmit power, the bigger is the performance difference.

\section{CONCLUSION}

This paper has studied the performance of the windowbased WIBA energy detector spectrum sensing method to complement the traditional database based spectrum sharing approaches for providing rural and remote area connectivity with $5 \mathrm{G}$ networks. Spectrum sensing can help in detecting unauthorized transmissions as well as facilitating the deployment of several secondary networks. Focus in this paper has been on assessing the achievable detection distance in kilometers which is critical for the establishment of remote area networks. The simulation results show that the WIBA method has excellent performance in a challenging rural area channel, and it clearly outperforms conventional energy detectors.

\section{REFERENCES}

[1] F. Hessar and S. Roy, "Capacity considerations for secondary networks in TV white space," IEEE Transactions on Mobile Computing, vol. 14, no. 9, 2015, pp. 1780-1793.

[2] Z. Huang, Y. Ma, Y. Li, and G. Wen, "A low complexity sub-optimal approach to dynamic spectrum allocation for white space devices with heterogeneous bandwidth requirements," IEEE Communications Letters, vol. 21 , no. 1,2017 , pp. 188-191.

[3] H. I. Volos and R. M. Buehrer, "Cognitive radio engine training," IEEE Transactions on Wireless Communications, vol. 11, no. 11, 2012, pp 3878-3889.

[4] P. L. M. Denkovska and L. Gavrilovska, "Geolocation database approach for secondary spectrum usage of TVWS," in 19thTelecommunications Forum (TELFOR), 2011, pp. 369-372.

[5] H. Saarnisaari and J. Vartiainen, "Spectrum window based signal detection at low SNR," in Int. Conf. on Military Communications and Information Systems (ICMCIS), Poland, Nov. 2018.

[6] Z. Khan, "Coordination and adaptation techniques for efficient resource utilization in cognitive radio networks," Ph.D. dissertation, Acta Univ Oul Technica C 395. Faculty of Technology, University of Oulu, Finland, Nov. 2011, http://urn.fi/urn:isbn:9789514295980 [retrieved: Jan, 2019].

[7] B. Sokal and et al, "D3.1 Physical layer of the 5G-RANGE Part I," 5G-RANGE Project, Tech. Rep., 2018.

[8] Ericsson and Telstra, "Measurements of Extreme Rural Scenarios," report R1-166599, October 2016, release 15.

[9] J. Proakis, Digital Communications. New York, USA: McGraw-Hill, 1995.

[10] H. Saarnisaari and P. Henttu, "Impulse detection and rejection methods for radio systems," in MILCOM, Boston, MA, USA, Oct. 2003.

[11] J. Vartiainen, J. J. Lehtomäki, and H. Saarnisaari, "Double-threshold based narrowband signal extraction," in VTC 2005, Stockholm, Sweden, May/June 2005, pp. 1288-1292.

[12] J. Vartiainen, "Concentrated signal extraction using consecutive mean excision algorithms," Ph.D. dissertation, Acta Univ Oul Technica C 368. Faculty of Technology, University of Oulu, Finland, Nov. 2010, http://jultika.oulu.fi/Record/isbn978-951-42-6349-1 [retrieved: Jan, 2016].

[13] H. Saarnisaari, P. Henttu, and M. Juntti, "Iterative multidimensional impulse detectors for communications based on the classical diagnostic methods," IEEE Trans. Commun., vol. 53, no. 3, Mar. 2005, pp. 395398.

[14] I. F. Akyildiz, B. F. Lo, and R. Balakrishnan, "Cooperative spectrum sensing in cognitive radio networks: A survey," Physical Communication, vol. 4, no. 1, 2011, pp. 40-62. 